\title{
Blue light therapy versus low dose isotretinoin in mild to moderate acne
}

\author{
Ayman Elgendy ${ }^{1 *}$, Karem Khali1 ${ }^{1}$, Eslam Alshawadfy ${ }^{2}$, Nancy Wadea ${ }^{1}$ and Osama Alkady ${ }^{1}$ \\ ${ }^{1}$ Department of Dermatology, Benha University, Egypt \\ ${ }^{2}$ Department of Dermatology Research, National Research Centre, Al bohouth St, Dokki, Cairo, Egypt
}

\begin{abstract}
Background: Despite advances in acne therapy, treatment failure is still common. Isotretinoin is the only drug that affects almost all factors in acne pathogenesis, however side-effects are common at the doses reported in published studies in the literature. Sun exposure is known to be beneficial for acne. Lasers/light-based devices may offer an alternative to conventional acne modalities in selected patients, such as non-responder or noncompliant patients or in antibiotic resistant patients. The aim of the study was to compare the efficacy of blue light and low dose isotretinoin in patients with mild to moderate acne.

Subjects and Methods: In this prospective comparative study, 60 consecutive patients with mild to moderate facial acne were recruited. They were randomized into 2 groups. The first group comprised 30 patients who were treated with blue light in two sessions weekly for 6 weeks. The second group comprised 30 patients who were treated with isotretinoin $0.3 \mathrm{mg} / \mathrm{kg} / \mathrm{d}$ for 6 months. No topical or other systemic therapy was allowed during the study period. Baseline grading of acne was done with Investigator's Global Assessment scale. Severity was assessed in each visit. Clinical photographs were obtained for evaluation every 4 weeks. Patients were followed up once every 2 weeks. However patients were asked to come earlier in case of any adverse events. Liver function tests (serum aspartate aminotransferaseAST, serum alanine transaminase ALT, direct and total bilirubin), and lipid profiles (total cholesterol, low-density lipoprotein, high-density lipoprotein, triglyceride) were evaluated for all patients, before the initiation of treatment and after one month of treatment.
\end{abstract}

Results: Patients in both groups revealed a reduction in the number of lesions. The first group showed a reduction of approximately $32 \%$ at 2 weeks and $76 \%$ at 6 weeks after 12 sessions blue light twice weeks for 6 weeks. The second group showed a reduction of approximately $15.3 \%$ at 2 weeks and $50 \%$ at 6 weeks after receiving $0.3 \mathrm{mg} / \mathrm{kg} / \mathrm{d}$ isotretinoin for 6 months.

Conclusion: Both blue light and low dose isotretinoin are effective in treating mild to moderate acne but blue light seems to be a more preferable choice being more safe, less expensive and of short treatment duration.

\section{Introduction}

Acne is a very common skin disease in young people, aged 1224 years old, but it can occur in anytime in life duration. This disease is a multifaceted disorder of the sebaceous glands [1]. Scientific understanding of the pathogenesis of acne has increased considerably. Acne vulgaris is traditionally managed with a variety of topical and systemic medications. Isotretinoin has been used more commonly in recent years, especially for treatment of nodular and nodulocystic acne. Oral isotretinoin is the single most effective agent for treatment of acne [2]. On the other hand; this agent is the only available drug that affects all four of the major pathogenic processes in acne. The downside of this drug is its toxicity [3]. Conventional dose of isotretinoin causes many side-effects such as teratogenicity, mucocutaneous events, increasing serum lipids and liver enzymes, gastrointestinal, ocular, and psychological side-effects [4]. For these reasons, low dose isotretinoin was tried in acne patients particularly mild to moderate cases.

Lasers/light-based devices may offer an alternative to conventional acne modalities in selected patients, such as non-responder or noncompliant patients or in antibiotic resistant patients [5]. This study was done to compare low dose isotretinoin versus blue light in treatment of mild to moderate acne patients.

\section{Subjects and methods}

\section{Patients}

In this prospective comparative study 60 patients with facial acne (mild, moderate) were enrolled. The patients were recruited from Al Dar Hospital Dermatology outpatient clinic over a period from February 2013 to December 2014. Acne severity grading was determined using Investigator's Global Assessment (IGA) scale. 30 patients were treated with blue light and the other 30 patients were treated with low dose isotretionin. Blue light group comprised 18 females and 12 males; ages ranged between 18 and 32 years. Low dose isotretinoin group included 16 females and 14 males with ages ranging between 16 and 28 years. The aim of this study was explained to patients. The females who were treated with isotretinoin were informed about risk of teratogenicity and the necessity to use two reliable simultaneous forms of contraception during treatment and for 3 months after the termination of treatment. The patients were on no medications for 4 weeks prior to the study. Each patient signed an informed consent.

Correspondence to: Ayman Elgendy, Professor of Dermatology and Venereology, Benha University, Egypt, Tel: +966507364687; E-mail: aymanelgendy91@yahoo.com

Key words: acne, bluelight, low dose, isotretinoin

Received: April 15, 2015; Accepted: May 22, 2015; Published: May 25, 2015 
Inclusion criteria were: patients of both sexes and of ages above 12 years and, patients having mild to moderate facial acne vulgaris who failed to respond to the classic topical treatment and patients willing to undergo treatment and follow ups.

Exclusion criteria for blue light therapy included the following: known light sensitivity; history of phototoxicity and history of herpes simplex virus or cold sores on the treatment area.

Exclusion criteria for isotretinoin therapy were patients with age less than 12 years, or those having severe facial acne vulgaris. Also pregnant women or who were planning to become pregnant during the course of treatment were excluded.

\section{Investigator's Global Assessment (IGA) scale}

\begin{tabular}{|c|c|}
\hline Score & Description \\
\hline 0 & No acne: No evidence of acne vulgaris \\
\hline 1 & $\begin{array}{l}\text { Minimal: Few non-inflammatory lesions (comedones) are } \\
\text { present; a few inflammatory lesions (papules/pustules) may } \\
\text { be present. }\end{array}$ \\
\hline 2 & $\begin{array}{l}\text { Mild: Several to many non-inflammatory lesions } \\
\text { (comedones) are present; a few inflammatory lesions } \\
\text { (comedones) are present }\end{array}$ \\
\hline 3 & $\begin{array}{l}\text { Moderate: Many non-inflammatory (comedones) } \\
\text { and inflammatory (papules/pustules) are present; no } \\
\text { nodulocystic lesions are present }\end{array}$ \\
\hline 4 & $\begin{array}{l}\text { Severe: Significant degree of inflammatory disease; papules/ } \\
\text { pustules are a predominant feature; a few nodulocystic } \\
\text { lesions may be present; comedones may be present }\end{array}$ \\
\hline
\end{tabular}

\section{Treatment protocol}

Blue light group, a high intensity, enhanced, narrowband, blue light source (cure light, Iclear XL) was used. This machine has a lamp emitting peak of (405-420 nm) for lesions. Treatment fluence used was $90 \mathrm{mw} / \mathrm{cm}^{2}$ of light. Each patient received treatment sessions twice a week for 6 weeks during which time any other acne therapy was prohibited. Each session was 30 minutes exposure time (15 minutes for each half of the face). During the treatment session, both eyes of the patient were protected with specific light protective goggles to prevent adverse effects.

Subjects were instructed to cleanse their face before each treatment with an unscented soap or nonirritant facial cleanser. They were also instructed to apply a moisturizing non-comedogenic sunscreen with Sun Protection Factor (SPF) 32 after each morning treatment as needed (for sun protection and to mitigate potential dryness and/or irritation).

In isotretinoin group, patients received doses at $0.3 \mathrm{mg} / \mathrm{kg} / \mathrm{d}$ in divided doses for six months. Liver function tests (serum aspartate aminotransferase AST, serum alanine transaminase ALT, direct and total bilirubin), and lipid profiles (total cholesterol, low-density lipoprotein, high-density lipoprotein, triglyceride) were evaluated for all patients, before the initiation of treatment and monthly thereafter. The criterion for discontinuation of isotretinoin therapy was a blood test rising above the following values in the first two months of follow-up: triglycerides $>400 \mathrm{mg} / \mathrm{dL}(4.52 \mathrm{mmol} / \mathrm{L})$, alkaline phosphatase $>264 /$ UL (female), $>500 / \mathrm{UL}$ (male), ALT>62/UL, AST $>80 / \mathrm{UL}$, cholesterol $>300 \mathrm{mg} / \mathrm{dL}$ (>7.7 mmol/L).

Clinical assessment was performed 6 times during treatment period $(0,2,6,10,16$ and 24 weeks). First criterion of assessment was counting the number of lesions (comedones, papules, pustules and total sum of the lesions). The second criterion was the Investigator's global improvement rating on a fine point scale $(-1=$ worsened, $0=$ unchanged, $1=$ improved, $2=$ markedly improved, and $3=$ resolved). Safety was assessed by asking patients about any symptoms of adverse reactions and laboratory changes especially in isotretinoin group. Patient satisfaction was determined regarding the line of treatment (satisfied or non satisfied). Clinical photographs were obtained for evaluation every 4 weeks.

\section{Statistics}

All data analyzed by Microsoft Office Excel 2007; in descriptive statics frequency (for qualitative data), mean, range, and standard deviation (for quantitative data), were used to demonstrate the results.

\section{Results}

Sixty patients were recruited for the study. Eight out of sixty (13.3\%) patients (3/30 patients of blue light group, 5/30 patients of isotretinoin group) discontinued treatment for non study-related reasons. In Blue light group, the numbers of comedones, papules, pustules, sum of all lesions were $(14+16.4,18+11.3,16+7.9,50+25.6$ respectively) before the treatment, and $(12+6.4,10+7.3,9+4.9,32+15.5$ respectively) after 2 weeks and $(8+6.4,4+7.3,3+4.9,12+9.3$ respectively) after 6 weeks. In isotretinoin group ,the numbers of comedones, papules, pustules, sum of all lesions were $(14+10.8,28+15.4,22+13.2,52+27.6$ respectively) before the treatment, $(10+8.4,18+6.4,10+7.3,9+4.9,44+15.6$ respectively) after 2 weeks and $(8+6.3,22+6.4,14+5.3,26+13.6$ respectively) after 6 weeks.

Safety was assessed during the study by the reporting of adverse events and laboratory changes. The most common side-effects reported in the present study were mild cheilitis in $17 / 25$ patients (68\%), mild generalized xerosis in $16 / 25$ patients (64\%) of isotretinoin group, and localized xerosis limited to treatment area (3/27) patients $(11.1 \%)$ of blue light group. The observed side-effects were mild. $2 / 25$ patients of isotretinoin group (8\%) showed elevated liver function test results, and increase in serum lipid profiles was mild. In none of the patients was the treatment discontinued due to the abnormality in laboratory values (Table 1 and Table 2).

Table 1. Physician's overall response rating.

\begin{tabular}{|c|c|c|c|c|}
\hline \multirow[t]{2}{*}{ Rating } & \multicolumn{2}{|c|}{ Blue light group } & \multicolumn{2}{|c|}{ Isotretinoin group } \\
\hline & Number & $(\%)$ & Number & $(\%)$ \\
\hline Discontinued & 3 & 10 & 5 & 16.6 \\
\hline Unchanged & 2 & 6.6 & 2 & 6.6 \\
\hline Improved & 5 & 16.6 & 3 & 10 \\
\hline Markedly improved & 16 & 53.3 & 14 & 46.6 \\
\hline Resolved & 4 & 13.3 & 6 & 20 \\
\hline Total & 30 & 100 & 30 & 100 \\
\hline
\end{tabular}

Table 2. Comparison between study groups.

\begin{tabular}{|l|l|l|}
\hline & Blue light group & Isotretinoin group \\
\hline Number & 30 & 30 \\
Sex & 18 females, 12 males & 16 females, 14 males \\
Discontinuation & 3 patients & 5 patients \\
Sum of lesions (mean \pm SD) & & \\
0 week & $50 \pm 25.6$ & $52 \pm 27.6$ \\
2 weeks & $32 \pm 15.5$ & $44 \pm 15.6$ \\
6 weeks & $12 \pm 9.3$ & $26 \pm 13.6$ \\
& Xerosis (affecting face(3/27) & -Cheilitis $(17 / 25)$, \\
Adverse effects & & -Xerosis affecting whole body $(16 / 25)$ \\
& $0 / 27$ & - Mild elevations $(2 / 25)$ \\
Laboratory changes & $22 / 27$ & $20 / 25$ \\
Patient satisfaction & & \\
\hline
\end{tabular}




\section{Discussion}

Acne is treated with a variety of topical and oral medications such as antibacterials, antimicrobials and retinoids. Conventional treatments for acne can be prolonged and associated with side-effects. Therefore, there is a growing demand by patients for a fast, safe, and side-effect-free therapy. Acne often improves after exposure to sunlight, and this observation has led to the development of laser and other light therapies. The conventionally used dose of isotretinoin (0.5$1 \mathrm{mg} / \mathrm{kg} / \mathrm{d}$ ) in acne causes significant dose-related adverse effects. To observe whether use of blue light or isotretinoin in low doses is more beneficial in treatment of mild to moderate acne, this comparative study was carried out.

Patients, who were treated with blue light sessions, showed early onset of clearance of acne lesions. The reduction of number of skin lesions was $32 \%$ at 2 weeks and $76 \%$ at 6 weeks. Patient satisfaction was 81.4\%; side effects were minimal in form of dryness $11.1 \%$. However with follow up at 3 months, few lesions reappeared $(22+11.6)$ in $18 / 27$ patients $(66.6 \%)$ which was cleared with use of topical retinoid, this indicates that for patients with mild to moderate acne we can start with blue light then maintenance with topical retinoid.

It was reported that the blue light device treatment is effective and well tolerated, offering rapid, gentle and convenient treatment of inflammatory acne, with the majority of subjects reporting that they were satisfied, very satisfied, or extremely satisfied with treatment. The blue light treatment is associated with significant reductions in the number, severity, and redness of flares and improvements in the skin's overall appearance as well as in clarity, radiance, tone, texture, and smoothness [6].

Blue light is commonly used to kill Propionibacterium acnes in clinics. Furthermore, blue light exhibits a good bactericidal effect against Helicobacter pylori, methicillin-resistant Staphylococcus aureus, Pseudomonas aeruginosa, and many oral bacteria in laboratory studies. The bactericidal mechanism of blue light as commonly accepted is through photo-excitation of intracellular porphyrins resulting in production of cytotoxic reactive oxygen species which can inactivate bacteria. Blue light can kill bacteria without additional exogenous photosensitizers [7].

There are several studies showing the efficacy of blue light, and combination of both blue and red light for the treatment of mild to moderate acne. Most of the studies were open label with sample size ranging between 30 to 50 patients. The results uniformly showed short term improvement in inflammatory lesions with blue light. Further none of the studies has showed follow up results beyond 12 weeks after treatment. Acne clearing is variable among patients and relapse rates are high after therapy is discontinued [8-10].

Isotretinoin is quite a useful therapeutic advance in the management of acne. The standard dose of isotretinoin is $0.5-1 \mathrm{mg} / \mathrm{kg} / \mathrm{day}$ for 20 24 weeks, with a maximum cumulative dose of $120-150 \mathrm{mg} / \mathrm{kg}$. It has been recommended for the treatment of severe nodulocystic acne in the doses of $1-2 \mathrm{mg} / \mathrm{day}$ [11]. While using this treatment protocol the incidence of side-effects is quite high and requires regular monitoring including a watch on the serum lipid profile. It has been debated whether isotretinoin should be reserved for severe nodulocystic acne only or it can be used for mild and moderate acne also. To decrease the incidence of side-effects and to make the therapy protocol simpler, the lower dose regimen has been tried by various authors. Most of these studies have found that low-dose and intermittent regimens of isotretinoin are effective in moderate to severe acne with a low incidence and severity of side-effects [12].

In the present study we have tried to improve the tolerability of isotretinoin therapy in acne patient by using a modified treatment regimen of low dose daily isotretinoin for 6 months. Onset of the improvement of acne lesions generally started 6 weeks after initiation of treatment (the reduction of number of skin lesions was 15.3\%), and was considered excellent at the end of treatment by all patients. Marked clinical improvement in inflammation, flattening of papulopustular lesions. Patient satisfaction was $80 \%$; side effects were minimal in form of cheilitis (68\%), xerosis (64\%) and mild elevation in lipid profile (8\%). However Rasi et al., 2014 reported that, the most common side-effects were mild cheilitis in 93 patients (66.4\%), mild xerosis in 12 patients $(8.5 \%)$.

Low dose isotretinoin to treat acne, has been tried previously. Isotretinoin administration for duration of 8.3 months in an initial dosage of $10 \mathrm{mg} /$ day up to $0.43 \mathrm{mg} / \mathrm{kg}$ produced very good results in $62.8 \%$ and good results in $31.9 \%$ of the patients [13]. After treating patients with 0.3 to $0.4 \mathrm{mg} / \mathrm{kg} /$ day dosage for 6 months, complete remission of $94.4 \%$ achieved in patients [4]. However, complete clearing in the present study was (92.5\%). The lower values in the present study are most likely due to the shorter treatment duration with healing of all lesions. Low dose isotretinoin represents a well-tolerated and efficient alternative to the standard $(0.5-1 \mathrm{mg} / \mathrm{kg} / \mathrm{day})$ route for treatment of mild to moderate acne. The only pitfall is the longer than duration of treatment period.

Another study reported that, at the end of isotretinoin treatment course, (96.4\%) demonstrated complete clearing of their acne. In 5-year follow-up, relapse occurred in 11 (7.9\%) of patients. All adverse effects were mild, and discontinuation of treatment was not necessary [14].

Isotretinoin at a dose of $20 \mathrm{mg} /$ day (approximately $0.3-0.4 \mathrm{mg} / \mathrm{kg}$ / day), for a period of 3 months resulted in very good improvement in $90 \%$ of participants. Cheilitis was the most common among the side effects observed and was seen in $98 \%$ of the participants. Elevated serum lipid levels were observed in $6 \%$ of the participants, and relapse occurred in $4 \%$ of the participants over a 6 month follow up period [15].

In conclusion, both blue light and low dose isotretinoin are effective in treatment of mild to moderate acne in patients who fail to respond to classic treatment but blue light therapy offers faster resolution and fewer side effects, better tolerance, less cost and less contraindications and hence more patient satisfaction. We also suggest combining blue light therapy with oral isotretinoin in treatment of acne to decrease dose and avoid the side effects of isotretinoin.

\section{References}

1. Dhir R, Gehi NP, Agarwal R, More YE (2008) Oral isotretinoin is as effective as a combination of oral isotretinoin and topical anti-acne agents in nodulocystic acne. Indian J Dermatol Venereol Leprol 74: 187. [Crossref]

2. Layton AM, Dreno B, Gollnick HP, Zouboulis CC (2006) A review of the European Directive for prescribing systemic isotretinoin for acne vulgaris. J Eur Acad Dermatol Venereol 20: 773-776. [Crossref]

3. Brelsford M, Beute TC (2008) Preventing and managing the side effects of isotretinoin. Semin Cutan Med Surg 27: 197-206. [Crossref]

4. Sundström A, Alfredsson L, Sjölin-Forsberg G, Gerdén B, Bergman U, et al. (2010) Association of suicide attempts with acne and treatment with isotretinoin: retrospective Swedish cohort study. BMJ 341: c5812. [Crossref]

5. Rai R, Natarajan K (2013) Laser and light based treatments of acne. Indian J Dermatol Venereol Leprol 79: 300-309. [Crossref] 
6. Wheeland RG, Koreck A (2012) Safety and Effectiveness of a New Blue Light Device for the Self-treatment of Mild-to-moderate Acne. J Clin Aesthet Dermatol 5: 25-31. [Crossref]

7. Wang C, Yang P, Wang N, Yao M2 (2014) Advances in the research on bactericidal effect of blue light and its mechanism. Zhonghua Shao Shang Za Zhi 30: 258-261. [Crossref]

8. Tzung TY, Wu KH, Huang ML (2004) Blue light phototherapy in the treatment of acne. Photodermatol Photoimmunol Photomed 20: 266-269. [Crossref]

9. Morton CA, Scholefield RD, Whitehurst C, Birch J (2005) An open study to determine the efficacy of blue light in the treatment of mild to moderate acne. J Dermatolog Treat 16: 219-223. [Crossref]

10. Lee SY, You CE, Park MY (2007) Blue and red light combination LED phototherapy for acne vulgaris in patients with skin phototype IV. Lasers Surg Med 39: 180-188. [Crossref]
11. Akman A, Durusoy C, Senturk M, Koc CK, Soyturk D, et al. (2007) Treatment of acne with intermittent and conventional isotretinoin: a randomized, controlled multicenter study. Arch Dermatol Res 299: 467-473. [Crossref]

12. Agarwal US, Besarwal RK, Bhola K (2011) Oral isotretinoin in different dose regimens for acne vulgaris: a randomized comparative trial. Indian J Dermatol Venereol Leprol 77: 688-694. [Crossref]

13. Hermes B, Praetel C, Henz BM (1998) Medium dose isotretinoin for the treatment of acne. J Eur Acad Dermatol Venereol 11: 117-121. [Crossref]

14. Rasi A, Behrangi E, Rohaninasab M, Nahad ZM (2014) Efficacy of fixed daily $20 \mathrm{mg}$ of isotretinoin in moderate to severe scar prone acne. Adv Biomed Res 3: 103. [Crossref]

15. Rao PK, Bhat RM, Nandakishore B, Dandakeri S, Martis J, et al. (2014) Safety and efficacy of low-dose isotretinoin in the treatment of moderate to severe acne vulgaris. Indian J Dermatol 59: 316. [Crossref]

Copyright: $(2015$ Elgendy A. This is an open-access article distributed under the terms of the Creative Commons Attribution License, which permits unrestricted use, distribution, and reproduction in any medium, provided the original author and source are credited. 